\title{
Hadron Spectroscopy
}

\section{Sasa Prelovsek*}

Faculty of Mathematics and Physics, University of Ljubljana, Jadranska 19, Ljubljana, Slovenia and Jozef Stefan Institute, Jamova 39, Ljubljana, Slovenia

E-mail: sasa.prelovsekeijs.si

Recent results on the hadron spectroscopy from lattice QCD are reviewed with emphasis on the meson sector and in particular on quarkonium-like $X Y Z$ states. I report on the first rigorous treatment of the near-threshold states $X(3872)$ and $D_{s}^{0}(2317)$, and the lattice searches for $Z_{c}^{+}(3900)$, $X(4140)$ and double-charm tetraquark states. Meson resonances in light, strange and charm sector are reviewed, where the resonances masses as well as the strong decay widths are reported. The first lattice QCD simulation of two coupled-channels is discussed.

The 32nd International Symposium on Lattice Field Theory,

23-28 June, 2014

Columbia University New York, NY

${ }^{*}$ Speaker. 


\section{Introduction}

I was requested to review the hadron spectroscopy focusing on mesons with particular emphasis on quarkonium-like states. Indeed, there is a compelling motivation at present to establish whether such hadrons arise from first-principle QCD or not. Several experiments have, for example, found resonant structures in the $J / \psi \pi^{+}$invariant mass, indicating the existence of hadrons with exotic flavor composition $\bar{c} c \bar{d} u$ (see [1] for review).

Therefore I will concentrate on the recent lattice results on the meson spectroscopy and separately discuss hadrons well-below strong decay threshold, those near threshold and resonances above threshold. Simulations of the exotic flavor channels will be reported, where I will provide recent results and also refer to some of the older simulations.

\section{The discrete spectrum from lattice and information encoded in it}

The physics information on a hadron (below, near or above threshold) is commonly extracted from the discrete energy spectrum in lattice QCD. The physical system for given quantum numbers is created from the vacuum $|\Omega\rangle$ using interpolator $\mathscr{O}_{j}^{\dagger}$ at time $t=0$ and the system propagates for time $t$ before being annihilated by $\mathscr{O}_{i}$. To study a meson state with given $J^{P}$ one can, for example, use $\mathscr{O} \simeq \bar{q} \Gamma_{q}, \quad\left(\bar{q} \Gamma_{1} q\right)_{\vec{p}_{1}}\left(\bar{q} \Gamma_{2} q\right)_{\vec{p}_{2}},\left[\bar{q} \Gamma_{1} \bar{q}\right]\left[q \Gamma_{2} q\right]$ with desired quantum numbers. After the spectral decomposition the correlators are expressed in terms of the energies $E_{n}$ of eigenstates $|n\rangle$ and their overlaps $Z_{j}^{n}$

$$
C_{i j}(t)=\left\langle\Omega\left|\mathscr{O}_{i}(t) \mathscr{O}_{j}^{\dagger}(0)\right| \Omega\right\rangle=\sum_{n} Z_{i}^{n} Z_{j}^{n *} e^{-E_{n} t}, \quad Z_{i}^{n} \equiv\left\langle\Omega\left|\mathscr{O}_{i}\right| n\right\rangle
$$

The most widely used method to extract $E_{n}$ and $Z_{i}^{n}$ from the correlation matrix $C_{i j}(t)$ is the generalized eigenvalue method $C(t) u^{n}(t)=\lambda^{n}(t) C\left(t_{0}\right) u^{n}(t)$ [2,3]. The energies $E_{n}$ are obtained from the exponential behavior of the eigenvalues $\lambda^{n}(t) \propto e^{-E_{n} t}$ at large $t$.

All physical eigenstates with given quantum numbers appear as energy levels in principle. These can be "one-hadron" states, "two-hadron" states and the multi-hadron states. In reality the eigenstates are mixtures of these Fock components. Three- and more-hadron states have not been taken into account in the actual simulations for the spectroscopy of the hadronic resonances yet ${ }^{1}$. The major step during the past few years came from treating two-hadron states rigorously. These have a discrete spectrum due to the periodic boundary condition on finite lattices. If the two hadrons do not interact, then the momenta of each hadron is $\vec{p}=\frac{2 \pi}{L} \vec{N}$ with $\vec{N} \in N^{3}$, and the non-interacting energies of $H_{1}(\vec{p}) H_{2}(-\vec{p})$ are $E^{n . i .}=E_{1}(p)+E_{2}(p)$ with $E_{1,2}(p)=\left(m_{1,2}^{2}+p^{2}\right)^{1 / 2}$. The energies $E_{n}$ extracted from the lattice are slightly shifted in presence of the interaction and the shift provides rigorous information on the scattering matrix, as discussed below. In experiment, two-hadron states correspond to the two-hadron decay products with a continuous energy spectrum.

Particular care has to be taken concerning the discretization errors related to the heavy quarks. It is comforting to see that the complementary methods lead to compatible results in the continuum limit. For hadrons containing the charm quarks it is common to compare $m-m_{\text {reference }}$ between lattice and experiment, where the leading discretization errors related to $m_{c}$ cancel.

\footnotetext{
${ }^{1}$ Multi-hadron states have been, for example, simulated to determine the binding energies of the nuclei.
} 
The evaluation of the correlation matrices including $\bar{q} q$ as well as $\bar{q} q \bar{q} q$ interpolating fields entails various Wick contractions, which often require quark propagators from any source to any sink location. These are not rendered by the conventional method, and all-to-all methods such as distillation [4], stochastic distillation [5] or noise reduction technique [6] are applied. These are based on a particular (separable) form for quark smearing and are particularly useful for hadron spectroscopy since the spectrum $E_{n}$ is independent of the shape for quark smearing.

All simulations of charmonia and other hidden charm channels reported here omit the charmquark annihilation contribution, while possible Wick contractions with $u / d / s$ annihilation are taken into account. The OZI-suppressed charm-annihilation represents mixing with channels that contain no charm quarks, and these decay channels are indeed suppressed or unobserved in experiment. The rigorous treatment of this Wick contraction presents an unsolved problem on the lattice due to the mixing with a number of light hadron channels and the noise in the charm disconnected diagrams.

\section{Information encoded in the finite-volume spectrum}

Most of the physics information on the hadron masses and strong interactions between them has been obtained from the finite volume spectrum $E_{n}$. The mass of a hadron well below strong decay threshold is simply $m=\left.E\right|_{\vec{p}=0}$. In the energy region near or above threshold the masses of bound-states and resonances have to be inferred from the infinite-volume scattering matrix of the one-channel (elastic) or multiple-channel (inelastic) scattering - I will refer to it as the rigorous treatment. The bound states correspond to poles of the scattering matrix at $p^{2}<0$ below threshold. The resonances masses and widths are derived from the Breit-Wigner type fits of the scattering matrix, cross-section or phase shift.

Three approaches for extracting the infinite-volume scattering matrix from the finite volume $E_{n}$ are employed in the lattice studies reported here. The methods were reviewed in more detail during plenary talks $[7,8]$ at this meeting:

1. The most widely used approach is based on Lüscher's seminal work $[9,10,11,12]$ and its generalizations. In the case of elastic scattering between two hadrons with zero-total momentum, the energy $E_{n}=\left(m_{1}^{2}+p^{2}\right)^{1 / 2}+\left(m_{2}^{2}+p^{2}\right)^{1 / 2}$ renders momenta $p$ of each meson in the region outside the interaction. The infinite volume phase shift at that energy is given by the Lüscher's relation $\delta_{l}(p)=\operatorname{atan}\left[\sqrt{\pi} p L / 2 Z_{00}\left(1 ;(p L / 2 \pi)^{2}\right)\right]$ if the partial wave $l$ dominates the scattering [12]. This is a favorable case where one equation determines one unknown $\delta_{l}\left(E_{n}\right)$ for each energy level $E_{n}$.

The generalizations of this relation to multiple partial waves, non-zero total momenta, twisted boundary conditions ${ }^{2}$, coupled-channel scattering and three-particle systems have also been derived in a series of papers recently (for reviews see [7, 8]). For each energy level $E_{n}$ this generally leads to one (determinant) equation with several unknown $\delta_{l}^{a}\left(E_{n}^{c m}\right)$ and the rigorous extraction becomes much more challenging. In this case the analysis may relay on certain parametrizations of the scattering matrix as a function of $E^{\mathrm{cm}}$, which may render the

\footnotetext{
${ }^{2}$ Partial twisting only in the valence sector represents an approximation if Lüscher's relations based on the full twisting are employed.
} 
otherwise unsolvable problem tractable. It is encouraging that the Hadron Spectrum Collaboration presented the first simulation of two-coupled channel system $K \pi-K \eta$ and extracted the poles corresponding to strange mesons relying on the parametrization of the scattering matrix [13].

2. The finite volume Hamiltonian Effective Field Theory (EFT) represents an analogous approach. The parameters of this theory are extracted by fitting the analytic expressions for the eigenvalues of the finite-volume EFT using the lattice spectrum $E_{n}[14,15]$.

3. HALQCD approach starts with the lattice determination of the Nambu-Bethe-Salpeter wave function for two hadrons as a function of their separation, and extracts the potential $V(r)$ between two hadrons from that [16]. The phase shifts for scattering are obtained by solving the Schrödinger equation for given $V(r)$. Note that the potential is not a physics observable, while the resulting phase shift is. It was shown that HALQCD approach gives results in agreement with Lüscher's approach for the $\pi \pi$ scattering with $I=2$ [17], while systematic comparisons for other channels have not been done yet. There are ongoing discussions as to whether this approach is as rigorous as the Lüscher-type approach. The method has recently been generalized to the case of multiple-channel scattering [18] and preliminary results for the coupled channels $\Lambda \Lambda-N \Xi-\Sigma \Sigma$ [19] are particularly valuable as treatment of such a problem using the Lüscher-type approach is very challenging.

The resulting phase shifts enable the determination of the masses for resonances and bound states. The simplest example is a one-channel elastic scattering in partial wave $l$, where the scattering matrix is parametrized in terms of the phase shift $\delta_{l}(p)$ and satisfies unitarity $S S^{\dagger}=1$

$$
S(p)=e^{2 i \delta_{l}(p)}, \quad S(p)=1+2 i T(p), \quad T(p)=\frac{1}{\cot \left(\delta_{l}(p)\right)-i}
$$

- In the vicinity of a hadronic resonance with mass $m_{R}$ and width $\Gamma$, the cross section $\sigma \propto$ $|T(p)|^{2}$ has a resonant shape with $\delta\left(s=m_{R}^{2}\right)=\frac{\pi}{2}$

$$
T(p)=\frac{-\sqrt{s} \Gamma(p)}{s-m_{R}^{2}+i \sqrt{s} \Gamma(p)}=\frac{1}{\cot \delta(p)-i}, \quad \Gamma(p)=g^{2} \frac{p^{2 l+1}}{s}, \quad \frac{p^{2 l+1}}{\sqrt{s}} \cot \delta(p)=\frac{1}{g^{2}}\left(m_{R}^{2}-s\right) .
$$

This is one possible Breit-Wigner parametrization which are all equivalent for small $\Gamma$. The width $\Gamma$ is parametrized in terms of the phase space and the coupling $g$ (3.2). The fit of $\delta_{l}(p)$ renders $m_{R}$ and $g$ or $\Gamma$. It is customary to compare $g$ rather than $\Gamma$ to experiment, since $\Gamma$ depends on the masses of the scattering particles (for example $m_{\pi}$ ) via the phase space.

- The bound state $(B)$ in $H_{1} H_{2}$ scattering is realized when $T(p)$ has a pole at $p_{B}^{2}<0$

$$
T=\frac{1}{\cot \left(\delta_{l}\left(p_{B}\right)\right)-i}=\infty, \cot \left(\delta\left(p_{B}\right)\right)=i, m_{B}=E_{H_{1}}\left(p_{B}\right)+E_{H_{2}}\left(p_{B}\right), p_{B}=i\left|p_{B}\right| .
$$

The location of an s-wave shallow bound state can be obtained by parametrizing $\delta_{0}$ near threshold using the effective range expansion and finding $p_{B}$ which satisfies $\cot \left(\delta\left(p_{B}\right)\right)=i$

$$
p \cot \left(\delta_{0}(p)\right)=\frac{1}{a_{0}}+\frac{1}{2} r_{0} p^{2}, \quad-\left|p_{B}\right|=\frac{1}{a_{0}}-\frac{1}{2} r_{0}\left|p_{B}\right|^{2} .
$$

The large negative scattering length $a_{0}$ indicates the presence of a shallow bound state [20]. 
So far I discussed physics information that is obtained from the finite volume spectrum $E_{n}$. The overlaps $Z_{i}^{n}=\left\langle\Omega\left|\mathscr{O}_{i}\right| n\right\rangle$ provide a wealth of information about the composition of each lattice eigenstate $|n\rangle$. They have been used so far mostly as a qualitative guidance on the importance of various Fock components. It remains an open question how to use this rich source of information to rigorously extract properties of the physical states, especially in the case of smeared quarks. Analytic considerations in this direction may prove fruitful.

\section{Hadrons well below threshold}

Well below strong decay threshold there are no multi-hadron states, and the mass of a single hadron is extracted from $m=\left.E\right|_{\vec{p}=0}$ extrapolated to $L \rightarrow \infty, a \rightarrow 0$ and $m_{q} \rightarrow m_{q}^{p h y s}$. Many precision results have been available for a number of years.

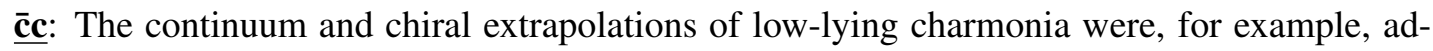
dressed by Fermilab/MILC [21] and HPQCD/MILC [22] collaborations at this meeting. The resulting splittings between the ground-state masses in different channels as well as the spin-averaged masses of $2 S$ and $1 S$ charmonia are in good agreement with experiment after extrapolations.

$\eta, \eta^{\prime}$ : These isosinglet pseudoscalar mesons can strongly decay only to the three-meson states, therefore they are very narrow and can be treated using standard technique to a good approximation. Their masses as well as the flavor mixing angle were determined as a function of $m_{\pi}$ by ETMC collaboration [23], recovering experimental values in the chiral limit.

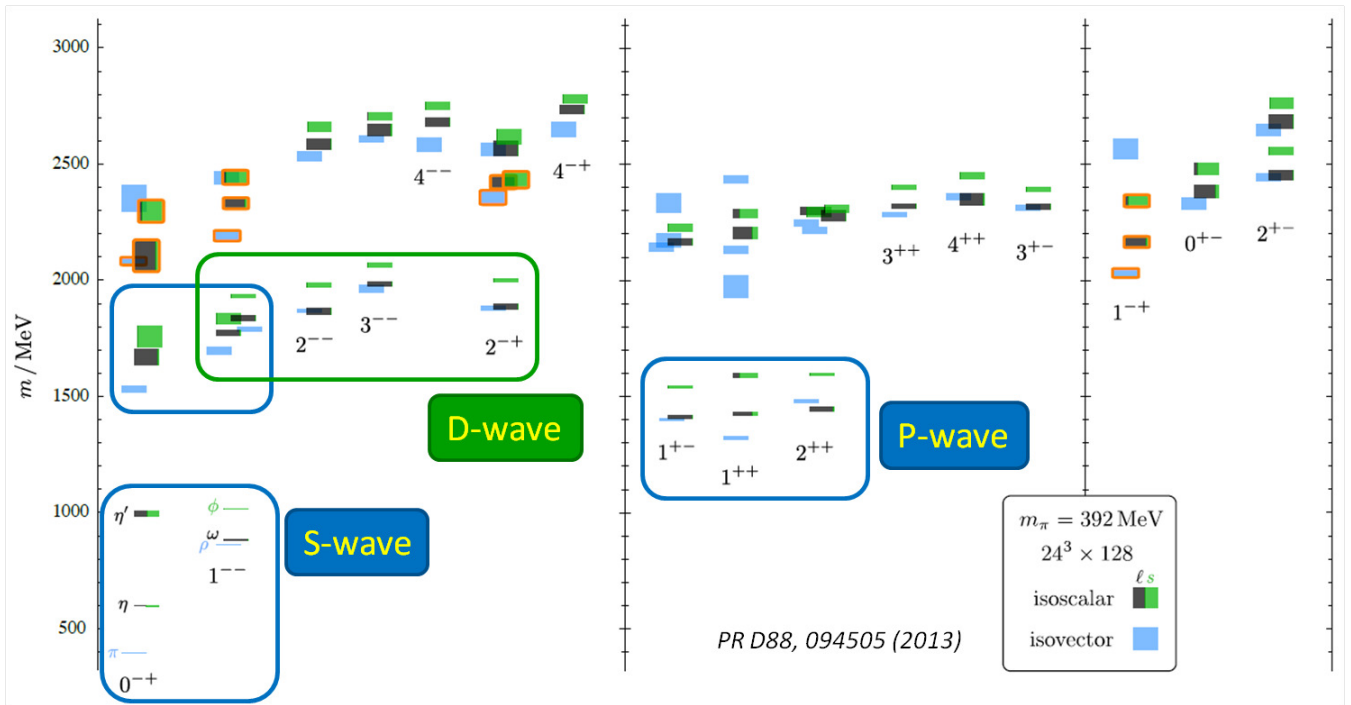

Figure 1: The isoscalar meson spectrum: black and green indicate percentage of $|l\rangle=(\bar{u} u+\bar{d} d) / \sqrt{2}$ and $|s\rangle=\bar{s}$ f for each state, respectively [24]. The blue levels show the masses of isovector mesons on the same ensemble. 


\section{Excited mesons within a single-hadron approach}

A great majority of hadrons lie near or above strong decay threshold. Yet most of them have been treated until recently based on a single-hadron approach. This entails (i) using only $\mathscr{O} \simeq \bar{q} q$ for mesons and $\mathscr{O} \simeq q q q$ for baryons, (ii) assuming that all energy levels correspond to "one-particle" states and (iii) that the mass of the excited resonance equals $m=E$. These are strong assumptions for the resonances, which are not asymptotic states. The approach also ignores the effect of the threshold on near-threshold states.

The most extensive light isoscalar [24], $D, D_{s}$ [25] and $\bar{c} c$ [26] spectra were extracted by the Hadron Spectrum Collaboration (HSC) on $N_{f}=2+1$ anisotropic configurations with $m_{\pi} \simeq$ $400 \mathrm{MeV}$ and two different $L \simeq 2.9 \mathrm{fm}, 1.9 \mathrm{fm}$. Their charmonium spectrum presents a valuable reference point for charmonium(like) states discussed later. The spectrum of isoscalar mesons as well as their light and strange content is shown in Fig. 1. An impressive number of excited states is extracted with a good accuracy in spite of the disconnected contribution for isoscalars. States are identified with members of $\bar{q} q$ multiplets $n S, n P$ and $n D$ based on the overlaps $\left\langle\mathscr{O}_{i} \mid n\right\rangle$, where interpolators are chosen to resemble multiplet members. The remaining states (indicated by orange) show particularly strong overlap with $\mathscr{O} \simeq \bar{q} F_{\mu \nu} q$ and are identified as hybrids.

\section{Near-threshold hadrons (beyond single-hadron approach)}

Most of the exciting states found by experiments are located near thresholds, for example $X(3872), Z_{c}^{+}(3900), Z_{b}^{+}(10610), Z_{b}^{+}(10650), D_{s}^{0}(2317)$ and $\Lambda(1405)$. The quarkonium-like states near threshold and above threshold are listed in Tables 10 and 12 of a valuable review by Brambilla et al. [1]. The lattice community is faced with an important challenge to establish whether these states arise from the first-principle QCD or not, and what is their nature.

Indeed, most of the effort in the hadron spectroscopy during past few years went in going beyond the single-hadron approach and taking into account two-hadron states rigorously. The majority of the studies focus on the (elastic) energy region near threshold, where the methods may be tractable at present, but one can not expect spectra of highly excited multiplets from the rigorous approach soon.

$\mathbf{Z}_{\mathbf{c}}^{+}$: Several charged-charmonia with quark content $\bar{c} c \bar{d} u$ were discovered recently in experiment. Most notably these are $Z^{+}(4430)$ with $I^{G}\left(J^{P C}\right)=1^{+}\left(1^{+-}\right)$discovered by Belle and confirmed by LHCb, and $Z_{c}^{+}(3900)$ with unknown $J^{P}$ discovered by BESIII and confirmed by Belle and CLEOc [1]. It is important to note that $Z_{c}^{+}(3900)$ was found in $J / \psi \pi$ invariant mass only through $e^{+} e^{-} \rightarrow Y(4260) \rightarrow\left(J / \psi \pi^{+}\right) \pi^{-}$. No resonant structure in $J / \psi \pi^{+}$was seen in $\bar{B}^{0} \rightarrow\left(J / \psi \pi^{+}\right) K^{-}$by BELLE [29], in $\bar{B}^{0} \rightarrow\left(J / \psi \pi^{+}\right) \pi^{-}$by LHCb [30] or in $\gamma p \rightarrow\left(J / \psi \pi^{+}\right) n$ by COMPASS [31]. This might indicate that the peak seen in $Y(4260)$ decay might not be of dynamical origin $[32,33]$.

The $Z^{+}$(4430) lies close to $D^{*} \bar{D}_{1}$ threshold and the only lattice search for it was performed in the quenched approximation soon after its discovery in 2007 [34]. The attraction between $D^{*}$ and $\bar{D}_{1}$ was found using Lüscher's approach, but no indication for a bound state. Further lattice studies were discouraged since this state lies above multiple thresholds. 


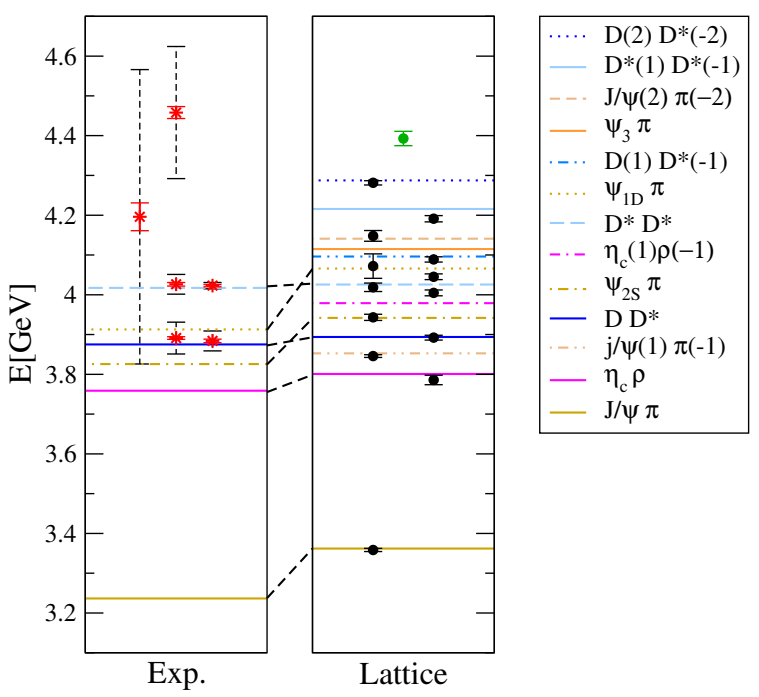

Figure 2: Left: The masses of the experimental $Z_{c}^{+} \simeq \bar{c} c \bar{d} u$ candidates [1] and the experimental thresholds. Right: The points represent the lattice spectrum $E_{n}$ in the $I^{G}\left(J^{P C}\right)=1^{+}\left(1^{+-}\right)$channel [27]. Lines represent the energies of the non-interacting two-meson states on this lattice. The states indicated by black circles are identified as two-meson states.

All lattice searches for $Z_{c}^{+}$considered $I^{G}\left(J^{P C}\right)=1^{+}\left(1^{+-}\right)$channel, which is the most relevant experimentally. The first lattice search for $Z_{c}^{+}(3900)$ considered $J / \psi \pi$ and $D \bar{D}^{*}$ scattering and only two-meson states $J / \psi \pi$ and $D \bar{D}^{*}$ were found, but no additional candidate for $Z_{c}^{+}$(3900) [35].

The $D \bar{D}^{*}$ interpolators were used to determine the s-wave and p-wave phase shift near $D \bar{D}^{*}$ threshold where experimental $Z_{c}(3900)$ is located. Partially twisted boundary conditions were applied taking into account s/p-wave mixing [36]. The authors conclude that no evidence for $Z_{c}^{+}(3900)$ is found. I would like to caution that $D \bar{D}^{*}$ correlators relax to the true ground state $e^{-\left(m_{J / \psi}+m_{\pi}\right) t}$ at large $t$ in [35], so the energies may not be reliable if only $D \bar{D}^{*}$ interpolators are employed.

The most extensive lattice search for $Z_{c}^{+}$with mass below $4.2 \mathrm{GeV}$ was performed in [27, 37]. The major challenge is presented by the two-meson states $J / \psi \pi, \psi_{2 S} \pi, \psi_{1 D} \pi, D \bar{D}^{*}, D^{*} \bar{D}^{*}$ and $\eta_{c} \rho$ that are inevitably present in the $I^{G}\left(J^{P C}\right)=1^{+}\left(1^{+-}\right)$channel, in addition to possible $Z_{c}^{+}$ candidates. Thirteen two-meson states are expected on the lattice with $L \simeq 2 \mathrm{fm}$ and $m_{\pi} \simeq 266 \mathrm{MeV}$ in the energy region of interest, and their non-interacting energies are shown by the horizontal lines in Fig. 2. The lattice spectrum of eigenstates is extracted using a number of meson-meson and $[\bar{c} \bar{d}]_{3_{c}}[\mathrm{cu}]_{\overline{3}_{c}}$ interpolating fields. Black circles show the lowest thirteen energy levels that are identified as two-meson states: they lie close to the non-interacting energies and show large overlap to the corresponding two-meson interpolators. No additional state that could represent $Z_{c}^{+}$is found below $4.2 \mathrm{GeV}$ [27]. The green state at $4.4 \mathrm{GeV}$ is not identified with $Z_{c}^{+}$since further two-meson states lie in this energy region.

A preliminary study that does not incorporate all low-lying two-mesons states around $4.2 \mathrm{GeV}$ seems to render a $Z_{c}^{+}$candidate at $4.16 \mathrm{GeV}[38]^{3}$, which is however not robust after further two-

\footnotetext{
${ }^{3}$ These preliminary results were reported at this meeting.
} 

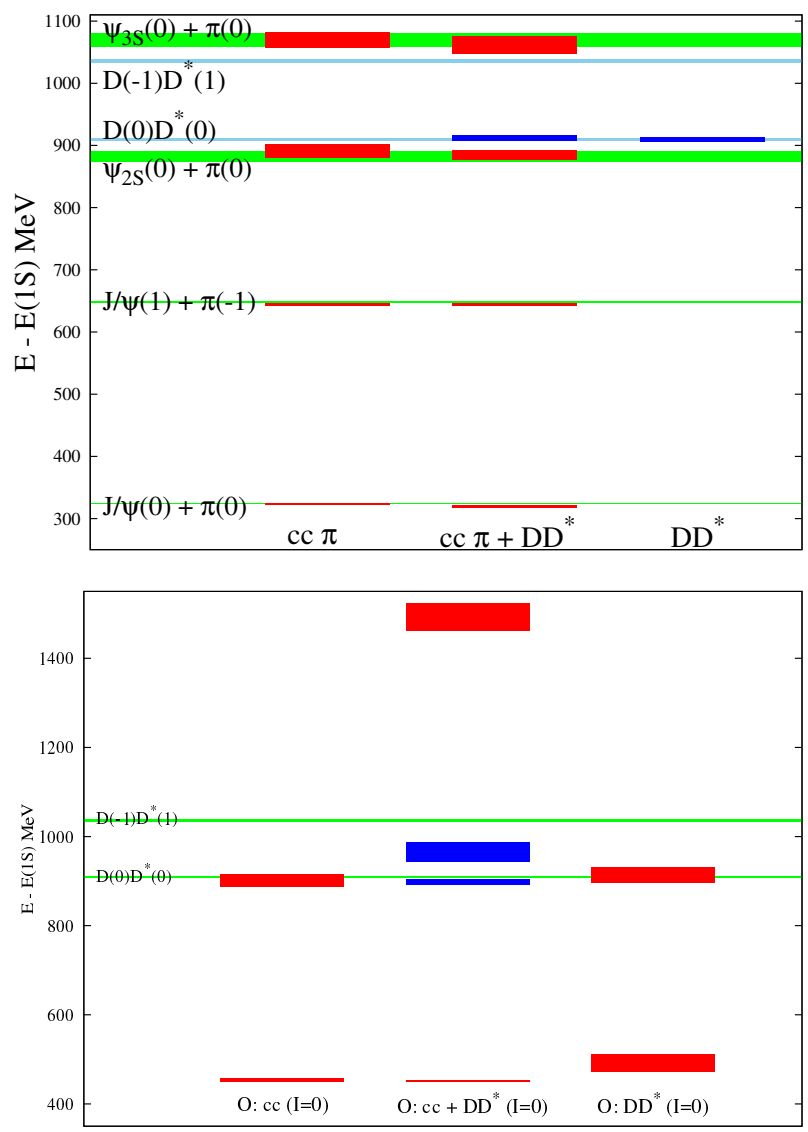

Figure 3: The spectra in the $Z_{c}^{+}$channel with $I\left(J^{P C}\right)=1\left(1^{+-}\right)$(upper pane), and in $X(3872)$ channel with $0\left(1^{++}\right)$(lower pane) [28]. The red and blue boxes are energies obtained from the simulation for various interpolator basis indicated at the bottom. The $c c$ and $c c \pi$ denote basis including charmonium and charmonium-pion interpolators, respectively. Lower pane: The lowest state is conventional $\chi_{c 1}$, the lower blue state is a candidate for $X(3872)$ with $I=0$, and the green lines denote non-interacting energies of $D \bar{D}^{*}$. Upper pane: the green levels denote non-interacting $\psi \pi$ states, while the light blue levels denote $D \bar{D}^{*}$. The state $D(1) \bar{D}^{*}(-1)$ is not seen in both channels [28].

meson states around 4.2 GeV are implemented [27]. Cautionary remarks based on this study are provided in Section V of [27].

The lattice simulation of the same channel based on the HISQ action gives the energies in Fig. 3 for various interpolator basis [28]. The figure illustrates that the two-meson states are seen if the corresponding interpolators are employed. The argument why $D(1) \bar{D}^{*}(-1)$ level does not appear is given in [28]. No additional state that could represent $Z_{c}^{+}$is found.

A large number of two-meson states apparently represents a major challenge in searching for $Z_{c}^{+}$or $Z_{b}^{+}$candidates using lattice QCD. Their spectra becomes denser with growing $L$, which poses a serious challenge already at $L \simeq 3 \mathrm{fm}$, as shown in Fig. 4 .

$\mathbf{X}(\mathbf{3 8 7 2})$ : The Belle discovery of neutral charmonium-like $X(3872)$ in 2013 initiated a new 

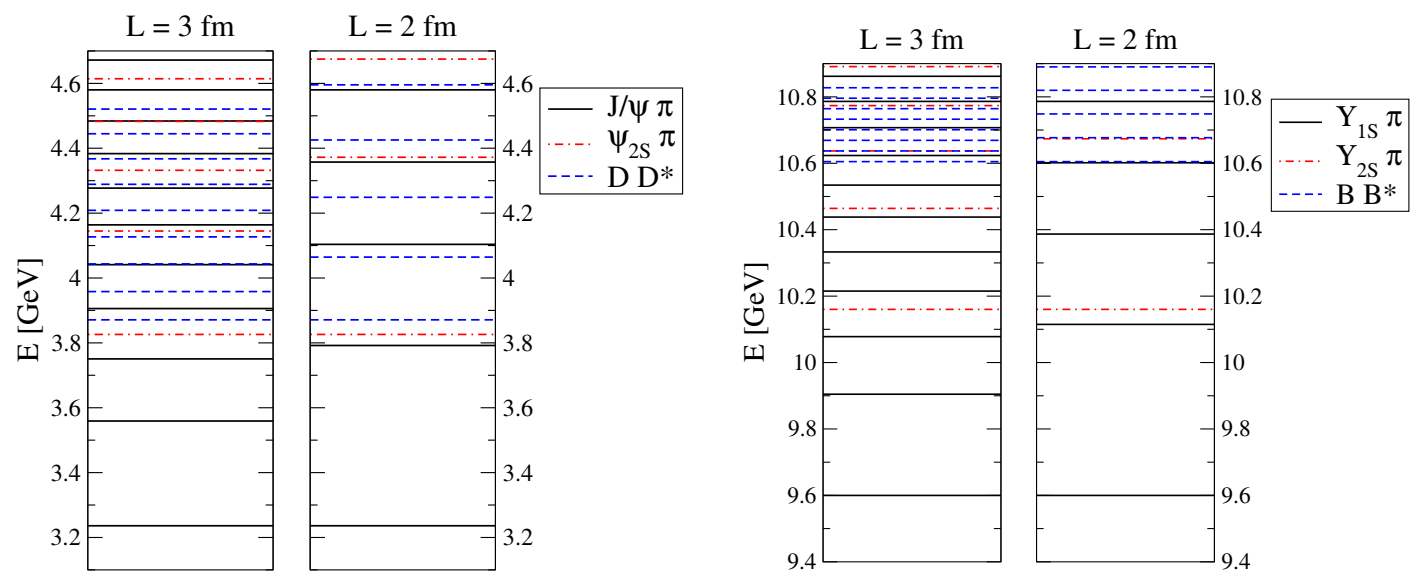

Figure 4: The energies of the non-interacting two-meson states for physical $m_{\pi}$ and $L=2,3$ fm in the $Z_{c}^{+}$(left) and $Z_{b}^{+}$(right) channels with $J^{P C}=1^{+-}$. Only three channels are shown and the spectrum is even denser when all channels are considered (see Fig. 2). Exotic experimental candidates are $Z_{b}^{+}(10610)$ and $Z_{b}^{+}(10650)$, while experimental $Z_{c}^{+}$candidates are shown in Fig. 2.

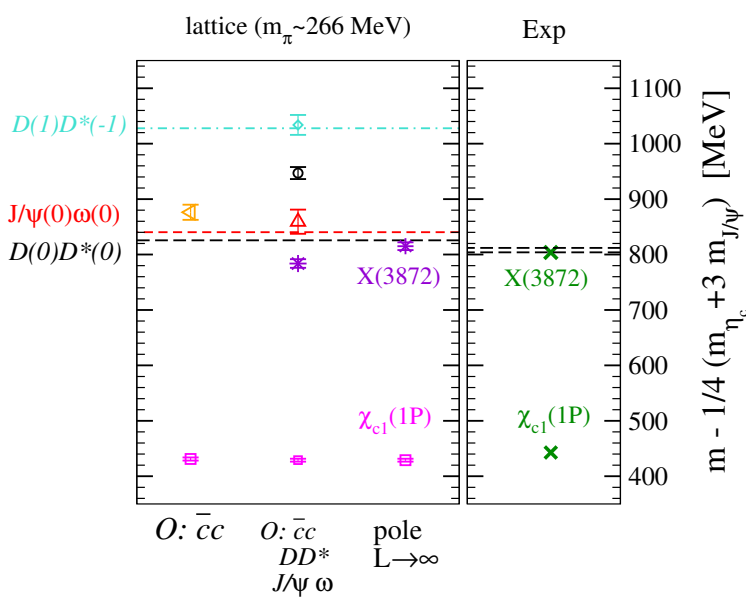

Figure 5: The spectrum in the $J^{P C}=1^{++}, I=0$ channel where charmonium-like $X(3872)$ resides: lattice results for two choices of the interpolator basis (points) and the energies of the non-interacting two-meson states (lines) [39]. The phase shift analysis renders the locations of the poles in $D \bar{D}^{*}$ scattering, which represent $\chi_{c 1}$ and $X(3872)$ bound states. These are close to the experimental masses, shown on the right.

era for unconventional-hadron spectroscopy. The state has $J^{P C}=1^{++}$, it is located within $1 \mathrm{MeV}$ of $D^{0} \bar{D}^{0 *}$ threshold and decays to $I=0$ as well as $I=1$ final states. The lattice simulations before 2013 used only $\bar{c} c$ interpolators and therefore ignored the effect of $D \bar{D}^{*}$ threshold. These typically rendered one energy level near $D \bar{D}^{*}$ threshold, but one could not reliably establish whether this state corresponds to $X(3872)$ or $D(0) \bar{D}^{*}(0)$.

A candidate for the charmonium(like) state $X(3872)$ was found $11 \pm 7 \mathrm{MeV}$ below the $D \bar{D}^{*}$ threshold for $J^{P C}=1^{++}, I=0, N_{f}=2$ and $m_{\pi} \simeq 266 \mathrm{MeV}$ [39] (see Fig. 5). This was the first lattice simulation that establishes a candidate for $X(3872)$ in addition to $\chi_{c 1}$ and the nearby scattering 

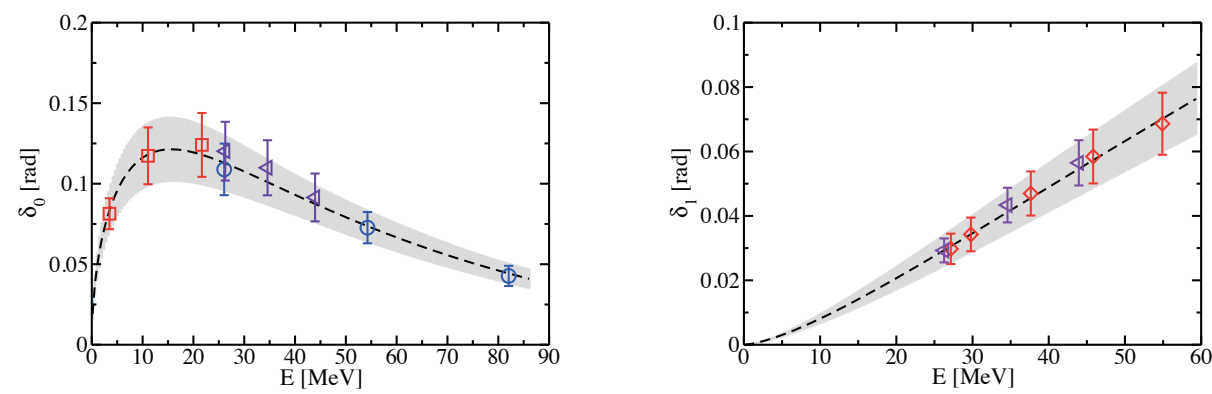

Figure 6: The s-wave and p-wave phase shift for $J / \psi \phi$ scattering aimed at searching for $X(4140)$ [43].

states $D \bar{D}^{*}$ and $J / \psi \omega$. The large and negative $a_{0}=-1.7 \pm 0.4 \mathrm{fm}$ for $D \bar{D}^{*}$ scattering length is one indication for a shallow bound state $X(3872)$, as can be understood from (3.4). The mass of the $X$ is determined from the position of the pole in $S$-matrix which is obtained by interpolating $D \bar{D}^{*}$ scattering phase shift near threshold $(3.3,3.4)$. The established $X(3872)$ has a large overlap with $\bar{c} c$ as well as $D \bar{D}^{*}$ interpolating fields; it is absent if one or the other type of interpolators are omitted. Only the $D \bar{D}^{*}$ and $J / \psi \rho$ scattering states are found in the $I=1$ channel, and there is no candidate for $X(3872)$ in this case [39]. This is in agreement with a popular interpretation that $X(3872)$ is dominantly $I=0$, while its small $I=1$ component arises solely from the isospin breaking and is therefore absent in the simulation with $m_{u}=m_{d}$.

New evidence for $X$ (3872) with $I=0$ came from the HISQ action at this meeting [28] and it is represented by the lower blue state in Fig. 3. The candidate for $X(3872)$ is found in the spectrum only if both $\bar{c} c$ and $D \bar{D}^{*}$ interpolators are used.

Recent analytic studies consider the quark mass dependence, the volume dependence and the effect from the isospin breaking relevant for future lattice studies of $X(3872)$ [40, 41, 42].

$\mathbf{X}(4140)$ : A structure called $X(4140)$ was found in the $J / \psi \phi$ invariant mass by CDF and more recently by CMS and D0, while its quantum numbers are unknown experimentally [1].

The s-wave and p-wave $J / \psi \phi$ scattering phase shift in Fig. 6 was extracted in a $N_{f}=2+1$ simulation using twisted boundary conditions [43], where mixing was taken into account. The $\bar{s} s$ annihilation contribution was omitted. The phase shifts do not support a resonant structure.

ccō̄ū tetraquarks: The potential $V(r)$ between $D=\bar{u} c$ and $D^{*}=\bar{d} c$ as a function of distance $r$ was extracted using the HALQCD time-dependent method [16]. The resulting potential in Fig. 7 is attractive, but the corresponding $D D^{*}$ scattering phase shift does not start at $\delta(0)=\pi$, which indicates that there is no $c c \bar{d} \bar{u}$ tetraquark bound state at the simulated $m_{\pi}$ [44].

No exotic candidates were seen also in a preliminary study of the double-charm tetraquark channel with $I=0$ and $I=1$ [45] that has been presented at this meeting.

$\mathbf{D}_{\mathbf{s} 0}^{*}, \mathbf{D}_{\mathbf{s 1}}$ : The quark models expected $D_{s 0}^{*}(2317)$ and $D_{s 1}(2460)$ above $D K$ and $D^{*} K$ thresholds, but they were experimentally found slightly below them. These are one of few shallow bound 

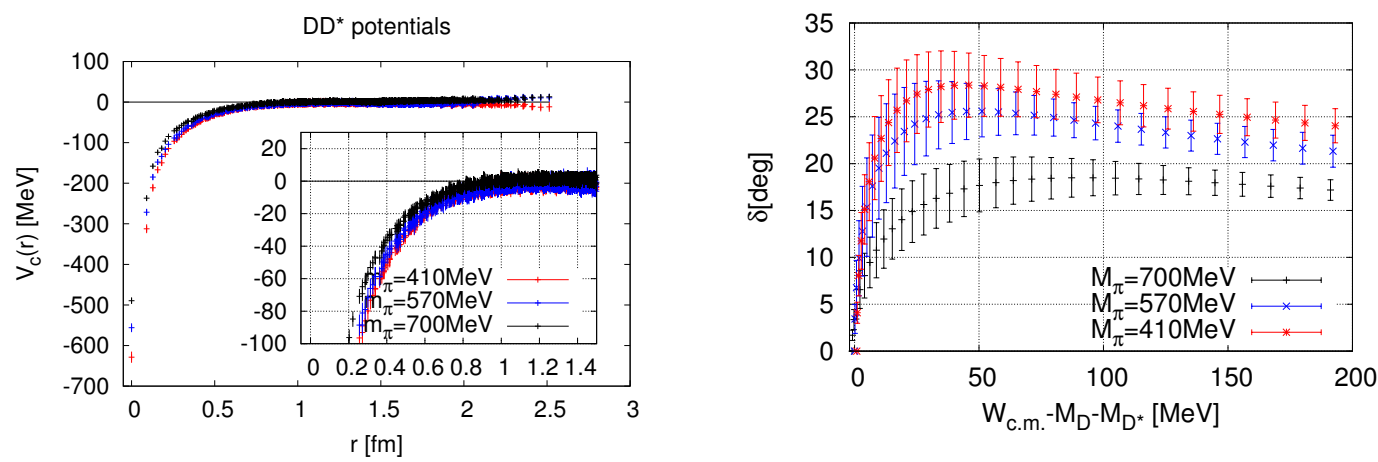

Figure 7: The potential $V(r)$ between $D$ and $D^{*}$ mesons as a function of distance $r$ calculated using HALQCD method, and the resulting $D D^{*}$ phase shift for this double-charm channel [44].

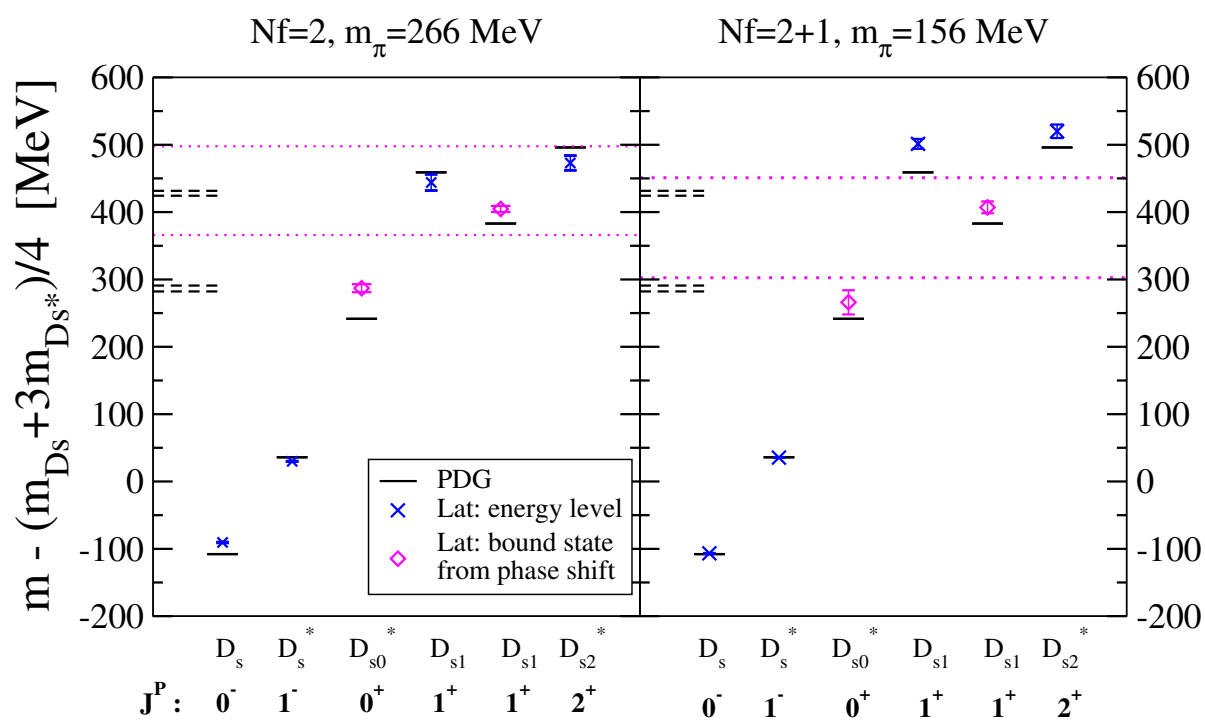

Figure 8: Spectra of $D_{s}$ mesons on two ensembles: the magenta points indicate masses inferred from poles in $D^{(*)} K$ scattering matrix; blue points are masses obtained using the single-hadron approach [46, 47, 48]. The back lines represent experimental masses.

states in the meson sector, and therefore deserve special attention. Many previous simulations extracted the ground states with $J^{P}=0^{+}$or $1^{+}$using $\bar{s} c$ interpolators, but one could not reliably establish whether these correspond to $D_{s 0} / D_{s 1}$ mesons or $D^{(*)}(0) K(0)$ states.

The first lattice simulation that takes the effect of these thresholds into account was performed during past two years $[46,47,48]$. It employs $D K$ and $D^{*} K$ interpolating fields in addition to the $\bar{s} c$. The position of thresholds is almost physical on $N_{f}=2+1$ ensemble with nearly physical $m_{\pi} \simeq$ $156 \mathrm{MeV}$. The $D^{(*)} K$ phase shift is extracted from each energy level and then parametrized in the region close to threshold using effective range formula (3.4). The scattering lengths are large and negative, which further supports the existence of the shallow bound states. Their binding momenta are obtained by searching for the pole in scattering matrix, which is realized at $\cot \left(\delta\left(p_{B}\right)\right)=i$ 


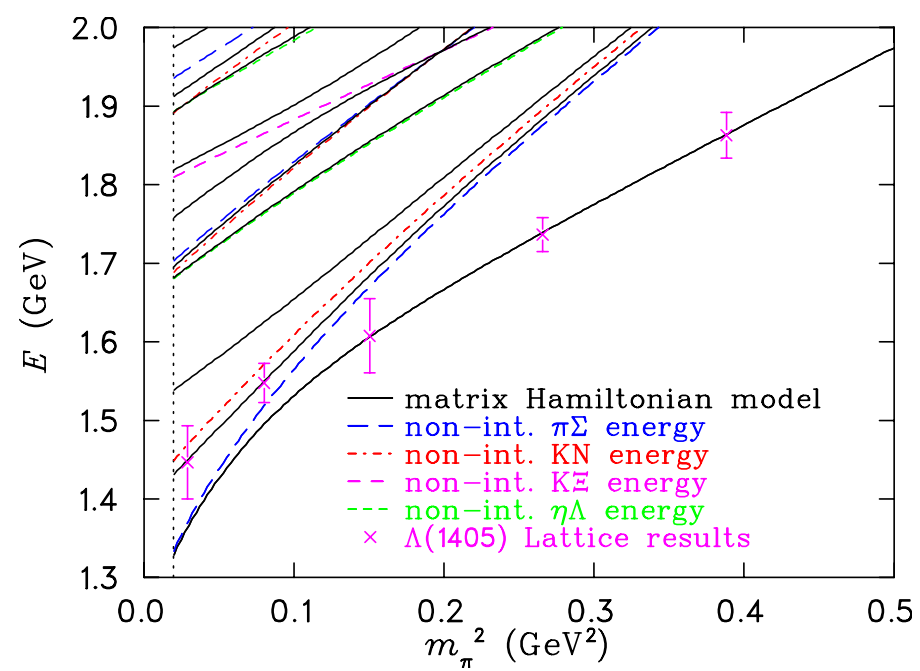

Figure 9: The study of $\Lambda(1405)$ channel: the lines represent the eigenvalues of the finite volume EFT for the coupled system of $u d s, \pi \Sigma, \bar{K} N, K \Xi$, which is fitted to the ground state energy obtained using $u d s$ interpolators (pink points) [15].

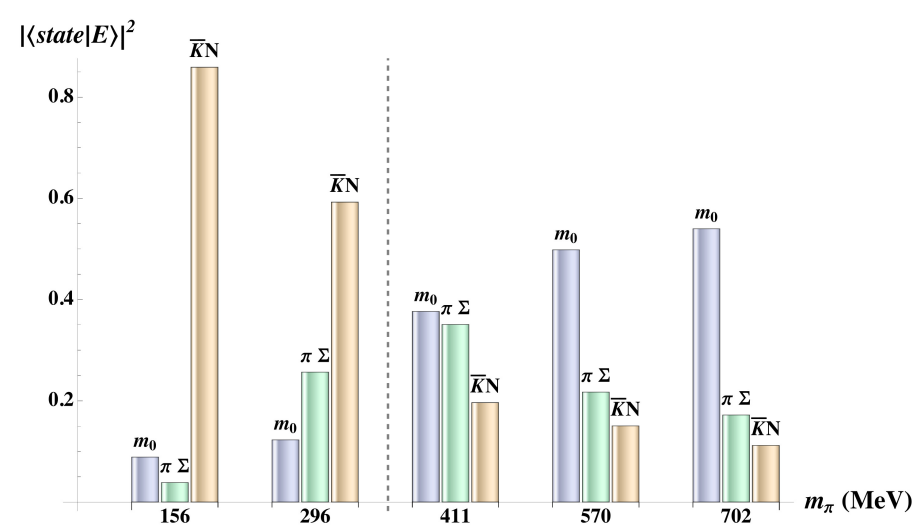

Figure 10: Composition of the $\Lambda(1405)$ eigenstate inferred from EFT in terms of various Fock components $u d s$ (denoted by $\left.m_{0}\right), \pi \Sigma$ and $\bar{K} N[15]$.

(3.3,3.4). These poles are related to $D_{s 0}^{*}(2317)$ and $D_{s 1}(2460)$ bound states and are close to the experimental masses. The summary of the resulting $D_{s}$ spectrum for these two as well as other $D_{s}$ states is shown in Fig. 8 for two values of pion masses.

A preliminary spectrum for these channels using $D K, \bar{s} c$ as well as $D_{s} \eta$ interpolating fields was presented at this conferences for zero and non-zero total momenta [49]. The goal is to determine the scattering matrix for the coupled channel system $D K-D_{s} \eta$, and look for signatures of bound states and resonances in it.

Baryon $\Lambda(\mathbf{1 4 0 5})$ : Experimentally, the $\Lambda(1405)$ baryon is a resonance in $\Sigma \pi$ scattering located slightly below $K N$ threshold.

The ground state energies for a range of $m_{\pi}$ (pink levels in Fig. 9) are obtained on PACS- 
CS ensembles using $\mathscr{O}=u d s$ and fitted with the eigenvalues of the Effective Hamiltonian Theory [15]. This effective theory describes the interaction between $u d s, \pi \Sigma, \bar{K} N, K \Xi$. Figure 9 shows the resulting eigenvalues of EFT (lines) and the composition of the $\Lambda(1405)$ eigenstate (histograms). This state is found to be dominated by $\bar{K} N$ Fock component at the lightest $m_{\pi}$. The conclusions of this study are based on the simulation without $\Sigma \pi$ and $\bar{K} N$ interpolators, and need to be verified with a simulation including them. Note that the ground state energy can get modified with the inclusion of two-hadron interpolators, for example in the case of a resonance [50, 51] or near-threshold state [46].

Preliminary results from including the baryon-meson interpolating fields in the correlation matrix for the spectroscopy of $\frac{1}{2}^{+}$nucleon channel where presented at this meeting [52, 53]. The first results in the $\frac{1}{2}^{-}$sector have been presented in [51].

\section{Rigorous treatment of hadronic resonances}

The rigorous treatment of a resonance in an elastic channel $M_{1} M_{2}$ amounts to a determination of the discrete spectrum including two-meson states, determination of the scattering phase shift from each energy level and making a Breit-Wigner type fit of the phase shift (3.2), as described in Section 2. The only hadron resonance studied in this way until recently is $\pi \pi \rightarrow \rho \rightarrow \pi \pi$, which has been simulated by a number of lattice collaborations until now (see for example [54]). In the following I summarize results for other channels, where only first pioneering steps have been made.

$\mathbf{K}^{*}, \kappa, \mathbf{K}_{\mathbf{0}}^{*}$ and $\mathbf{K}_{\mathbf{2}}$ : $K^{*}$ mesons and in particular the $K^{*}(892)$ were frequently addressed in lattice simulations, but always ignoring that the $K^{*}(892)$ decays strongly.

The $K \pi$ system with total momenta $P=\frac{2 \pi}{L} e_{z}, \frac{2 \pi}{L}\left(e_{x}+e_{y}\right)$ and 0 was simulated in [55], allowing the extraction of the phase shift for p-wave scattering with $I=1 / 2$. A Breit-Wigner fit of the phase in Fig. $11 \mathrm{~b}$ renders a $K^{*}(892)$ resonance mass $m^{\text {lat }}=891 \pm 14 \mathrm{MeV}$ and the $K^{*}(892) \rightarrow K \pi$ coupling $\tilde{g}^{l a t}=5.7 \pm 1.6$ compared to the experimental values $m^{\text {exp }} \approx 892 \mathrm{MeV}$ and $\tilde{g}^{\exp }=5.72 \pm 0.06$, where $\tilde{g}$ parametrizes the $K^{*} \rightarrow K \pi$ width $(3.2)^{4}$. Mixing of $p$ and $d$ wave is taken into account when extracting the phase shift around the $K^{*}(1410)$ and $K_{2}^{*}(1430)$ resonances. The $K^{*}(1410)$ resonance mass is found at $m^{\text {lat }}=1.33 \pm 0.02 \mathrm{GeV}$ compared to $m^{\text {exp }}=$ $1.414 \pm 0.0015 \mathrm{GeV}$ assuming the experimental $K^{*}(1410) \rightarrow K \pi$ coupling.

The first simulation of two-coupled channel system $K \pi-K \eta$ was presented just before this meeting $[13,56]$. The scattering matrix in the complex plane was parametrized as a function of $E^{c m}$ and the parameters were extracted using the fit to the finite volume spectrum via Lüscher-type method. The phase shifts $\delta^{K \pi}, \delta^{K \eta}$ and the inelasticity $\eta$ corresponding to the scattering matrix $T_{i i} \propto \eta e^{2 i \delta_{i}(p)}-1$ are shown in Fig. 11a. The poles related to $\kappa$ and $K^{*}(892)$ are found below $K \pi$ threshold for the employed $m_{\pi} \simeq 400 \mathrm{MeV}$, while $K_{0}^{*}(1430)$ and $K_{2}$ are found as resonances above threshold (see Fig. 11c).

$\mathbf{D}_{\mathbf{0}}^{*}$ and $\mathbf{D}_{\mathbf{1}}$ : The first rigorous simulation of a hadronic resonance that contains charm quarks addresses the broad scalar $D_{0}^{*}(2400)$ and the axial $D_{1}(2430)$ charmed-light mesons [50]. These

\footnotetext{
${ }^{4}$ The couplings $\tilde{g}$ and $g(3.2)$ are related by $g^{2}=\tilde{g}^{2} /(6 \pi)$.
} 
(a)

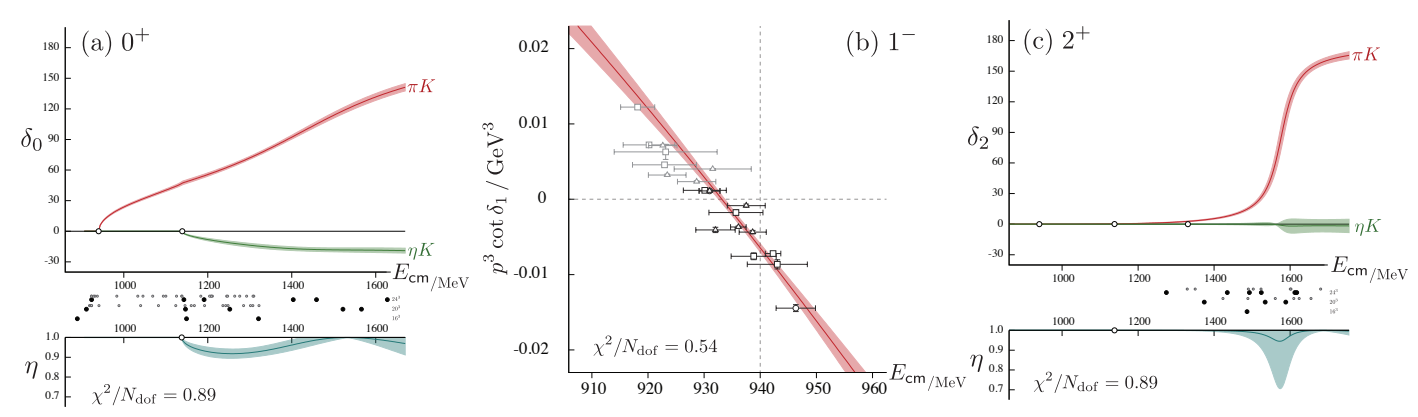

(b)

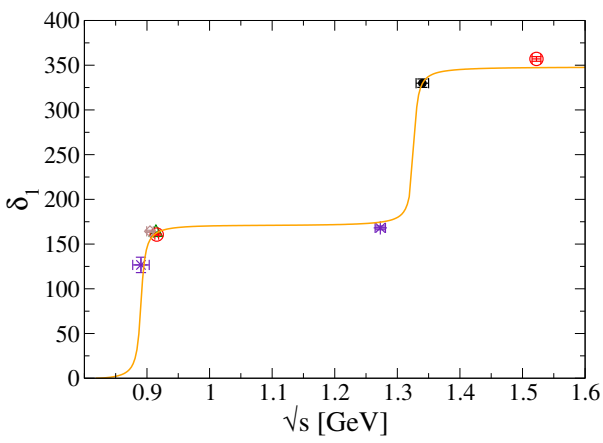

(c)

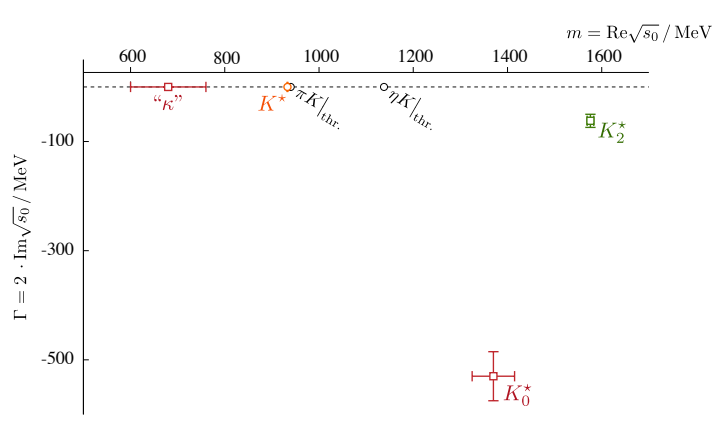

Figure 11: (b) $\delta_{l=1}^{K \pi}$ phase shift from [55]; (a) $\delta^{K \pi}, \delta^{K \eta}$ and the inelasticity $\eta$ from [13, 56]; (c) the poles of the scattering matrix in the complex plane correspond to the strange mesons $[13,56]$.

appear in s-wave scattering for $D \pi$ and $D^{*} \pi$, respectively. The resonance parameters are obtained using a Breit-Wigner fit to the elastic phase shifts at $N_{f}=2$ and $m_{\pi} \simeq 266 \mathrm{MeV}$. The resulting $D_{0}^{*}(2400)$ mass is $351 \pm 21 \mathrm{MeV}$ above the spin-average $\frac{1}{4}\left(m_{D}+3 m_{D^{*}}\right)$, in agreement with the experimental value of $347 \pm 29 \mathrm{MeV}$. The resulting $D_{0}^{*} \rightarrow D \pi$ coupling $g^{\text {lat }}=2.55 \pm 0.21 \mathrm{GeV}$ is close to the experimental value $g^{\text {exp }} \leq 1.92 \pm 0.14 \mathrm{GeV}$, where $g$ parametrizes the width (3.2). The results for $D_{1}(2430)$ are also found close to the experimental values; these are obtained by appealing to the heavy quark limit, where the neighboring resonance $D_{1}(2420)$ is narrow. These resonance masses are compared to the experimental ones in Fig. 12. The plot displays also the spectra for other charmed mesons obtained within single-hadron approach.

It is puzzling why the strange $D_{s 0}^{*}(2317)$ and the non-strange $D_{0}^{*}(2400)$ charmed scalar mesons have a mass within $1 \mathrm{MeV}$ of each other experimentally, while one would naively expect a larger slitting $O\left(m_{s}\right)$. The question is whether this near degeneracy is due to the strange meson being pushed down or the non-strange one being pushed up. This puzzle can be addressed by considering the lattice results for $D_{0}^{*}(2400)$ [50], which is found as a resonance in $D \pi$, and $D_{s 0}^{*}(2317)$ [46], which is found as a pole below $D K$. Both masses are found close to experiment. This favors the interpretation that the near degeneracy is a consequence of strange meson being pushed down due to $D K$ threshold. On the other hand, the interpretation that $D_{0}^{*}(2400)$ is pushed up due to tetraquark Fock component $\bar{u} \bar{s} s c$ is disfavored since $N_{f}=2$ simulation [50] renders its mass close to the experiment without any strange content in the valence or sea sectors.

A preliminary spectrum for $I=1 / 2$ channel channel using $D \pi, \bar{u} c$ as well as $D \eta$ interpolating 


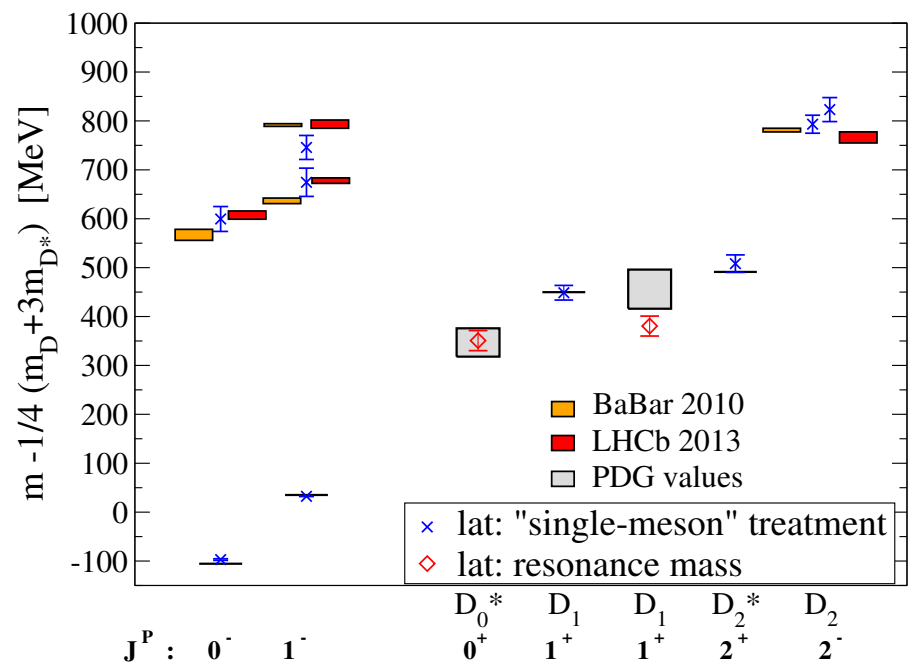

Figure 12: The spectrum of $D$ mesons: lattice results for the masses in $0^{+}$and $1^{+}$channels are obtained using the Breit-Wigner fit of $D^{(*)} \pi$ phase shift (red diamonds); other masses rely on the single hadron approach (blue crosses) [50]. BaBar and more recent LHCb results are shown side-by-side.

fields was presented at this meeting [49]. The coupled-channel analysis to extract the scattering parameters and resonances is planned.

$\underline{\mathbf{a}_{\mathbf{1}} \text { and } \mathbf{b}_{\mathbf{1}}}$ : The light axial-vector resonances $a_{1}(1260)$ and $b_{1}(1235)$ were explored for $N_{f}=2$ by simulating the corresponding scattering channels $\rho \pi$ and $\omega \pi$ [57]. Interpolating fields $\bar{q} q$ and $\rho \pi$ or $\omega \pi$ were used to extract the $s$-wave phase shifts for the first time. It is assumed that $\rho$ and $\omega$ are stable, which is justified in the energy region of interest for the employed parameters $m_{\pi} \simeq 266 \mathrm{MeV}$ and $L \simeq 2 \mathrm{fm} \mathrm{[58].} \mathrm{A} \mathrm{Breit-Wigner} \mathrm{fit} \mathrm{of} \mathrm{the} s$-phase shift gives the $a_{1}(1260)$ resonance mass $m_{a_{1}}^{\text {res }}=1.435(53)\left({ }_{-109}^{+0}\right) \mathrm{GeV}$ compared to $m_{a_{1}}^{\exp }=1.230(40) \mathrm{GeV}$. The $a_{1}$ width is parametrized in terms of the coupling $g$ (3.2), which results in $g=1.71(39) \mathrm{GeV}$ compared to $g^{\exp }=1.35(30) \mathrm{GeV}$ derived from experiment.

The analytic framework for the scattering of unstable particles in the finite volume was recently formulated in [58]. The approach was applied to study the effect of the $\rho \rightarrow \pi \pi$ decay to the $\rho \pi$ scattering, and this is found to be negligible for $L$ and $m_{\pi}$ employed in [57].

Light isovectors: An extensive study of various light isovector channels was performed with a number of $\bar{q} q$ and two-meson interpolators $\pi \pi, \eta \pi, \phi \pi, \bar{K} K$ in [59]. The effective masses of 16th till 31st eigenstate in the $\rho$-meson channel are shown in Fig 13 to demonstrate the high statistic quality of these high-lying states, obtained using the stochastic distillation. Energies of the lowest fifty eigenstates are also provided, together with their dominant Fock components based on $Z_{i}^{n}$. The lowest level, for example, is related to the $\rho$-meson.

\section{Related topics}

Mass decomposition of mesons: The meson mass $M$ is decomposed to the quark mass part 

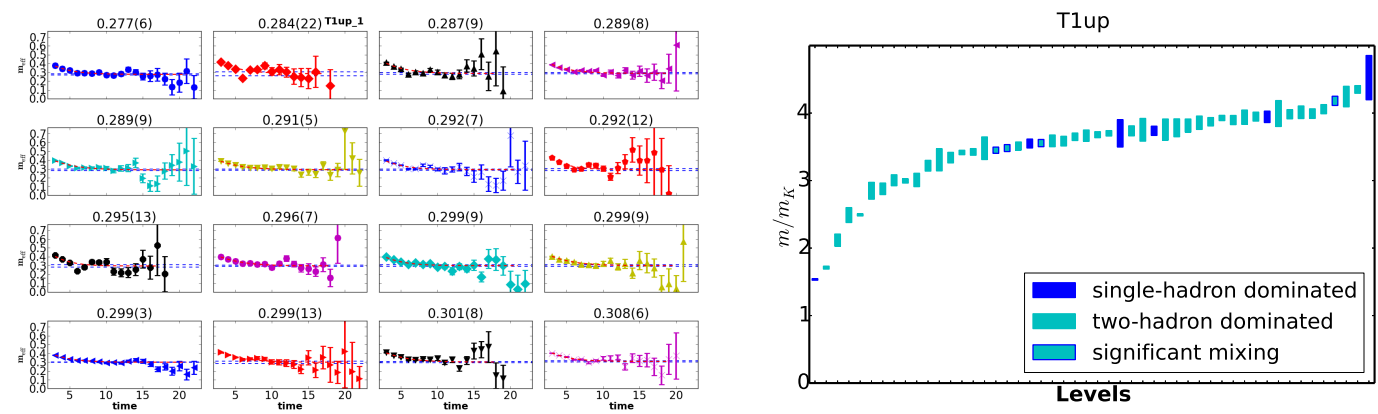

Figure 13: Left: The effective masses of the 16th excited to the 31st excited state in the $\rho$ channel obtained using a number of $\bar{q} q$ and meson-meson interpolators [59]. Right: The energies of the lowest 50 eigenstates in the same channel indicating their dominant Fock components.

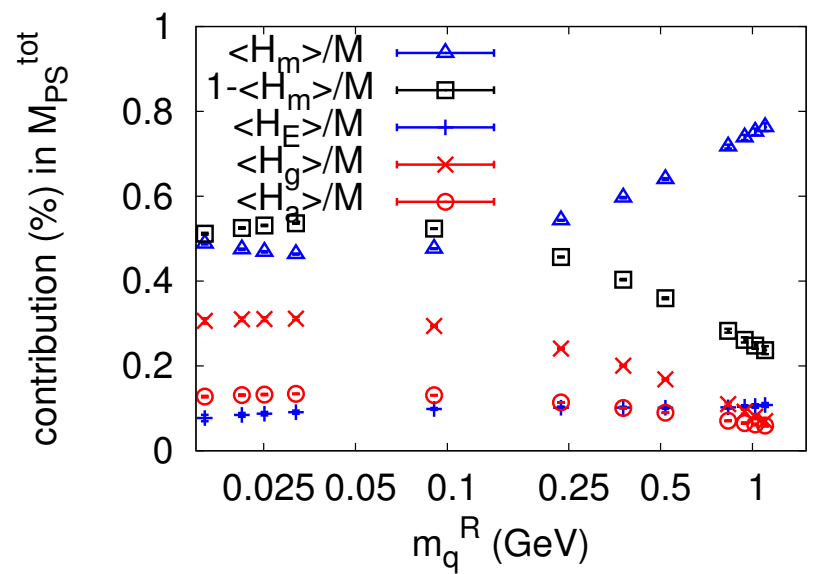

Figure 14: The percentage of various contributions (defined in the main text) to the pseudoscalar meson mass as a function of the quark mass $[60,61]$.

$H_{m}$, the quark energy part $H_{E}$, the gluon field energy part $H_{g}$ and the QCD trace anomaly part $H_{a}$, i.e. $M=-\left\langle T_{44}\right\rangle=\left\langle H_{E}\right\rangle+\left\langle H_{m}\right\rangle+\left\langle H_{g}\right\rangle+\left\langle H_{a}\right\rangle$ [60, 61]. Furthermore, these can be related via $\frac{1}{4} M=\frac{1}{4}\left\langle H_{m}\right\rangle+\left\langle H_{a}\right\rangle$. The $M,\left\langle H_{E}\right\rangle$ and $\left\langle H_{m}\right\rangle$ are determined through the two- and three-point correlators directly on the lattice, while $\left\langle H_{g}\right\rangle$ and $\left\langle H_{a}\right\rangle$ are obtained from the analytic relations presented in this paragraph. Proportions of each contribution to the pseudoscalar meson mass as a function of the quark mass is presented in Fig. 14. For results on the vector meson and interesting physics conclusions, I refer to [60, 61].

Extended QCD: The QCD is reformulated as the so-called Extended-QCD by the help of additional bosonic fields, where the second theory is exactly equivalent to QCD [62]. It is shown that the $N_{c} \rightarrow \infty$ limit of the Extended-QCD respects two properties that were difficult to reconcile in the past: (i) it makes direct connection to the constituent quark model with the constituent quark mass, (ii) it respects chiral symmetry rendering massless pion in the continuum limit. The 
approach may prove valuable to understand why quark models often work well even beyond the $N_{c} \rightarrow \infty$ limit.

\section{Conclusions}

Spectra of hadrons below strong decay thresholds are calculated with unprecedented accuracy. Extensive results for multiplets within the single-hadron approach are available in light and heavy, meson and baryon sectors. The first rigorous treatment of interesting near-threshold mesons $X(3872), D_{s 0}(2317)$ and $D_{s 1}(2460)$ was performed during the past two years. They appear as poles of the scattering matrix and their masses are close to the measured ones. Until recently, $\rho$ was the only resonance that was extracted using a Breit-Wigner type fit of the scattering phase shift, and it remains the only one that is mapped out in a great precision and by a number of collaborations. Strange, charmed and light-axial meson resonances have been rigorously treated for the first time recently. The first simulation of the two-coupled channel system was performed this year, where the poles corresponding to the strange mesons were extracted relying on the parametrization of the scattering matrix. Let me note that hadrons listed in this paragraph do not have manifestly exotic flavor and lattice QCD indeed presents convincing support for them.

On the other hand, we have no reliable lattice evidence for flavor exotic states yet, in spite of the number of searches. These have thoroughly looked for possible $\bar{c} c \bar{d} u$ candidates with $J^{P C}=1^{+-}$ in the vicinity of $D \bar{D}^{*}$ threshold, where $Z_{c}^{+}(3900)$ was found experimentally. No $\bar{c} c \bar{s} s$ resonance was found in $J / \psi \phi$ scattering on the lattice, and no $c c \bar{d} \bar{u}$ bound state in the $D D^{*}$ scattering.

However, there are many possibilities for improving searches of quarkonium-like states and other exotic states on the lattice. The available simulations present only the first steps in this direction. The lattice community is welcome to respond to the challenge set by the recent exciting experimental discoveries. This seems realistic for the states relatively near the strong decay thresholds, while much more challenging for higher lying states.

\section{Acknowledgements}

I would like to thank to Christine Davies, Carleton DeTar, Derek Leinweber, Keh-Fei Liu, Daniel Mohler, Colin Morningstar, Raul Briceno, Takeshi Yamazaki, Andrea Guerrieri, Christopher Thomas and David Wilson for valuable discussions and material related to this review talk. I am grateful to Christian B. Lang, Luka Leskovec, Daniel Mohler and Richard Woloshyn for the pleasure of collaborating on the described topics, and for reading this manuscript. This work is supported by ARRS project number N1-0020 and FWF project number I1313-N27.

\section{References}

[1] N. Brambilla, S. Eidelman, P. Foka, S. Gardner, A. Kronfeld, et al., QCD and Strongly Coupled Gauge Theories: Challenges and Perspectives, [arXiv: 1404.3723 ].

[2] C. Michael, Adjoint Sources in Lattice Gauge Theory, Nucl. Phys. B 259 (1985) 58.

[3] B. Blossier, M. DellaMorte, G. von Hippel, T. Mendes, and R. Sommer, On the generalized eigenvalue method for energies and matrix elements in lattice field theory, JHEP 0904 (2009) 094, [arXiv:0902.1265]. 
[4] Hadron Spectrum Collaboration, M. Peardon, J. Bulava, J. Foley, C. Morningstar, J. Dudek, R. G. Edwards, B. Joo, H.-W. Lin, D. G. Richards, and K. J. Juge, A novel quark-field creation operator construction for hadronic physics in lattice QCD, Phys. Rev. D 80 (2009) 054506, [arXiv:0905.2160].

[5] C. Morningstar, J. Bulava, J. Foley, K. J. Juge, D. Lenkner, M. Peardon, and C. H. Wong, Improved stochastic estimation of quark propagation with Laplacian Heaviside smearing in lattice QCD, Phys. Rev. D 83 (2011) 114505, [arXiv: 1104 . 3870].

[6] G. Bali, S. Collins, and A. Schafer, Effective noise reduction techniques for disconnected loops in Lattice QCD, [arXiv:0910.3970].

[7] T. Yamazaki, Hadronic Interaction, PoS LATTICE2014 (2014) 009.

[8] R. Briceno, Few-body physics, PoS LATTICE2014 (2014) 008.

[9] M. Lüscher, Volume dependence of the energy spectrum in massive quantum field theories. I. Stable particle states, Commun. Math. Phys. 104 (1986) 177.

[10] M. Lüscher, Volume dependence of the energy spectrum in massive quantum field theories. II. Scattering states, Commun. Math. Phys. 105 (1986) 153.

[11] M. Lüscher, Two-Particle States on a Torus and Their Relation to the ScatteringMatrix, Nucl. Phys. B 354 (1991) 531.

[12] M. Lüscher, Signatures of unstable particles in finite volume, Nucl. Phys. B 364 (1991) 237.

[13] J. J. Dudek, R. G. Edwards, C. E. Thomas, and D. J. Wilson, Resonances in coupled $\pi K, \eta K$ scattering from quantum chromodynamics, [arXiv:1406.4158].

[14] J. Hall, A. C. P. Hsu, D. Leinweber, A. Thomas, and R. Young, Finite-volume matrix Hamiltonian model for a $\Delta \rightarrow N \pi$ system, Phys.Rev. D87 (2013) 094510, [arXiv:1303.4157].

[15] D. Leinweber and et al., The Lambda 1405 is an anti-kaon-nucleon molecule, PoS LATTICE2014 (2014) 094.

[16] HAL QCD Collaboration, N. Ishii et al., Hadron-Hadron Interactions from Imaginary-time Nambu-Bethe-Salpeter Wave Function on the Lattice, Phys.Lett. B712 (2012) 437, [arXiv:1203.3642].

[17] HAL QCD Collaboration, B. Charron, A comparative study of two lattice approaches to two-body systems, PoS LATTICE2013 (2013) 223, [arXiv: 1312 . 1032].

[18] S. Aoki, B. Charron, T. Doi, T. Hatsuda, T. Inoue, et al., Construction of energy-independent potentials above inelastic thresholds in quantum field theories, Phys.Rev. D87 (2013), 034512, [arXiv:1212.4896].

[19] HAL QCD Collaboration, K. Sasaki, Quark mass dependence of hyperonic interactions from lattice $Q C D, P o S$ LATTICE2012 (2012) 157.

[20] S. Sasaki and T. Yamazaki, Signatures of S-wave bound-state formation in finite volume, Phys.Rev. D74 (2006) 114507, [hep-lat/0610081].

[21] D. Mohler, Low lying charmonium states at the physical point, PoS LATTICE2014 (2014) 086.

[22] B. Galloway, C. Davies, C. DeTar, P. Knecht, and J. Koponen, Radial and orbital excitation energies of charmonium, PoS LATTICE2014 (2014) 092. 
[23] ETM Collaboration, C. Michael, K. Ottnad, and C. Urbach, $\eta$ and $\eta^{\prime}$ mixing from Lattice $Q C D$, Phys.Rev.Lett. 111 (2013) 181602, [arXiv: 1310.1207$].$

[24] Hadron Spectrum Collaboration, J. J. Dudek, R. G. Edwards, P. Guo, and C. E. Thomas, Toward the excited isoscalar meson spectrum from lattice QCD, Phys.Rev. D88 (2013) 094505, [arXiv:1309.2608].

[25] G. Moir, M. Peardon, S. M. Ryan, C. E. Thomas, and L. Liu, Excited spectroscopy of charmed mesons from lattice QCD, JHEP 1305 (2013) 021, [arXiv: 1301 . 7670 ].

[26] Hadron Spectrum Collaboration, L. Liu et al., Excited and exotic charmonium spectroscopy from lattice QCD, JHEP 1207 (2012) 126, [arXiv: 1204.5425$].$

[27] S. Prelovsek, C. B. Lang, L. Leskovec, and D. Mohler, Study of the $Z_{c}^{+}$channel using lattice QCD, [arXiv:1405.7623v2].

[28] C. DeTar and S. Lee, The charmonium states X(3872)( $\left.1^{++}\right)$and Zc(3900) $\left(1^{+-}\right)$on HISQ lattices, PoS LATTICE2014 (2014) 125.

[29] Belle Collaboration, K. Chilikin et al., Observation of a new charged charmonium-like state in $B \rightarrow J / \psi K \pi$ decays, [arXiv:1408.6457].

[30] LHCb Collaboration, R. Aaij et al., Measurement of the resonant and CP components in $\bar{B}^{0} \rightarrow J / \psi \pi^{+} \pi^{-}$decays, Phys.Rev. D90 (2014) 012003, [arXiv: 1404.5673 ].

[31] COMPASS Collaboration, C. Adolph et al., Search for exclusive photoproduction of $Z_{c}^{ \pm}(3900)$ at COMPASS, [arXiv:1407.6186].

[32] D.-Y. Chen, X. Liu, and T. Matsuki, Reproducing the $Z_{c}(3900)$ structure through the initial-single-pion-emission mechanism, Phys.Rev. D88 (2013) 036008, [arXiv: 1304.5845 ].

[33] E. Swanson, Coupled Channel Cusps and $Z_{b}(10610), Z_{b}(10650)$, and $Z_{c}(3900)$, [arXiv:1409.3291].

[34] CLQCD Collaboration, G.-Z. Meng et al., Low-energy $D^{*+} D_{1}^{0}$ Scattering and the Resonance-like Structure $Z^{+}$(4430), Phys.Rev. D80 (2009) 034503, [arXiv: 0905 . 0752].

[35] S. Prelovsek and L. Leskovec, Search for $Z_{c}^{+}(3900)$ in the $1^{+-}$Channel on the Lattice, Phys.Lett. B727 (2013) 172-176, [arXiv:1308.2097].

[36] Y. Chen, M. Gong, Y.-H. Lei, N. Li, J. Liang, et al., Low-energy Scattering of $\left(D \bar{D}^{*}\right)^{ \pm}$System And the Resonance-like Structure $Z_{c}(3900)$, [arXiv:1403.1318].

[37] L. Leskovec, C. B. Lang, D. Mohler, and S. Prelovsek, Study of the $Z_{c}^{+}$channel using lattice QCD, PoS LATTICE2014 (2014) 118, , [arXiv: 1410 . 8828].

[38] S. Prelovsek, C. B. Lang, L. Leskovec, and D. Mohler, Evidence for a charged charmonium-like $Z_{c}^{+}$ from $Q C D,[a r X i v: 1405.7623 v 1]$.

[39] S. Prelovsek and L. Leskovec, Evidence for X(3872) from DD* scattering on the lattice, Phys.Rev.Lett. 111 (2013) 192001, [arXiv:1307.5172].

[40] M. Jansen, H.-W. Hammer, and Y. Jia, Light quark mass dependence of the X(3872) in an effective field theory, Phys.Rev. D89 (2014) 014033, [arXiv:1310.6937].

[41] E. Garzon, R. Molina, A. Hosaka, and E. Oset, Strategies for an accurate determination of the X(3872) energy from QCD lattice simulations, Phys.Rev. D89 (2014) 014504, [arXiv:1310.0972]. 
[42] M. Albaladejo, C. Hidalgo-Duque, J. Nieves, and E. Oset, Hidden charm molecules in a Finite Volume, Int.J.Mod.Phys.Conf.Ser. 26 (2014) 1460059, [arXiv: 1312.5339 ].

[43] S. Ozaki and S. Sasaki, Lúscher's finite size method with twisted boundary conditions: an application to $J / \psi-\phi$ system to search for narrow resonance, Phys.Rev. D87 (2013) 014506, [arXiv:1211.5512].

[44] Y. Ikeda, B. Charron, S. Aoki, T. Doi, T. Hatsuda, et al., Charmed tetraquarks $T_{c c}$ and $T_{c s}$ from dynamical lattice QCD simulations, Phys.Lett. B729 (2014) 85-90, [arXiv:1311.6214].

[45] A. Guerrieri, Flavored tetraquark spectroscopy, PoS LATTICE2014 (2014) 106.

[46] D. Mohler, C. B. Lang, L. Leskovec, S. Prelovsek, and R. Woloshyn, $D_{s 0}^{*}(2317)$ Meson and D-Meson-Kaon Scattering from Lattice QCD, Phys.Rev.Lett. 111 (2013) 222001, [arXiv:1308.3175].

[47] C. B. Lang, L. Leskovec, D. Mohler, S. Prelovsek, and R. Woloshyn, $D_{s}$ mesons with $D K$ and $D^{*} K$ scattering near threshold, Phys.Rev. D90 (2014) 034510, [arXiv: 1403.8103 ].

[48] C. B. Lang, L. Leskovec, D. Mohler, and R. Woloshyn, DK and $D^{*} K$ scattering near threshold, PoS LATTICE2014 (2014) 086, [arXiv: 1410.6668 ].

[49] S. Ryan, A study of scattering in open charm channels, PoS LATTICE2014 (2014) 114.

[50] D. Mohler, S. Prelovsek, and R. Woloshyn, D $\pi$ scattering and D meson resonances from lattice QCD, Phys.Rev. D87 (2013) 034501, [arXiv:1208.4059].

[51] C. B. Lang and V. Verduci, Scattering in the $\pi N$ negative parity channel in lattice QCD, Phys.Rev. D87 (2013) 054502, [arXiv:1212.5055].

[52] W. Kamleh, A. Kiratidis, and D. Leinweber, Nucleon spectroscopy using multi-particle operators, PoS LATTICE2014 (2014) 098.

[53] V. Verduci, Baryon resonances coupled to Pion-Nucleon states in lattice QCD, PoS LATTICE2014 (2014) 121.

[54] J. J. Dudek, R. G. Edwards, and C. E. Thomas, Energy dependence of the $\rho$ resonance in $\pi \pi$ elastic scattering from lattice QCD, Phys.Rev. D87 (2013) 034505, [arXiv: 1212 . 0830].

[55] S. Prelovsek, L. Leskovec, C. B. Lang, and D. Mohler, $K \pi$ scattering and the $K^{*}$ decay width from lattice QCD, Phys.Rev. D88 (2013) 054508, [arXiv: 1307.0736 ].

[56] D. Wilson, Resonances in $\pi K$ scattering, PoS LATTICE2014 (2014) 081.

[57] C. B. Lang, L. Leskovec, D. Mohler, and S. Prelovsek, Axial resonances $a_{1}(1260), b_{1}(1235)$ and their decays from the lattice, JHEP 04 (2014) 162, [arXiv: 1401.2088 ].

[58] L. Roca and E. Oset, Scattering of unstable particles in a finite volume: the case of $\pi \rho$ scattering and the $a_{1}(1260)$ resonance, Phys.Rev. D85 (2012) 054507, [arXiv: 1201.0438 ].

[59] C. Morningstar and et al., Excited isovector mesons using the stochastic LapH method, PoS LATTICE2014 (2014) 101, [arXiv:1410.8839].

[60] Y.-B. Yang, Y. Chen, T. Draper, M. Gong, K.-F. Liu, et al., Meson Mass Decomposition from Lattice $Q C D$, [arXiv:1405.4440].

[61] L. Liu, Search for $Z_{c}$ (3900) on the lattice with twisted mass fermions, PoS LATTICE2014 (2014) 117.

[62] D. B. Kaplan, Extended QCD, [arXiv:1306.5818]. 